\title{
Bone enhancing effect of titanium-binding proteins isolated from bovine bone and implanted into rat calvaria with titanium scaffold
}

\author{
Yoshinori Kuboki ${ }^{\mathrm{a}, * * *}$, Toshitake Furusawa** ${ }^{\text {b }}$, Masaaki Sato ${ }^{\mathrm{b}}$, Yongkun Sun ${ }^{\mathrm{c}}$, \\ Hidero Unuma $^{\mathrm{d}}$, Shigeaki Abe ${ }^{\mathrm{e}}$, Ryuichi Fujisawa ${ }^{\mathrm{e}}$, Tsukasa Akasaka ${ }^{\mathrm{e}}$, Fumio Watari ${ }^{\mathrm{e}}$, \\ Hiroko Takita ${ }^{\mathrm{e}}$ and Rachel Sammons ${ }^{\mathrm{f}}$ \\ a Professor Emeritus, Hokkaido University, Sapporo, Japan \\ ${ }^{\mathrm{b}}$ Graduate School of Bio-Medical Engineering, Tohoku University, Sendai, Japan \\ ${ }^{\mathrm{c}}$ Graduate School of Environmental Science, Hokkaido University, Sapporo, Japan \\ ${ }^{\mathrm{d}}$ Graduate School of Science and Engineering, Yamagata University, Yamagata, Japan \\ ${ }^{\mathrm{e}}$ Graduate School of Dental Medicine, Hokkaido University, Sapporo, Japan \\ ${ }^{\mathrm{f}}$ School of Dentistry, Birmingham University, Birmingham, UK
}

Received 3 December 2012

Accepted 29 August 2013

\begin{abstract}
Based on our previous finding that a chromatography with titanium beads selectively binds phosphoproteins, including caseins, phosvitin and dentin phosphoproteins, we investigated whether bone phosphoproteins also bind to titanium. Bovine bone matrix proteins were extracted with $2 \mathrm{M}$ urea/PBS after demineralization. The $2 \mathrm{M}$ urea extract was directly applied to the titanium chromatography column as reported. The chromatogram showed an initial large peak at breakthrough position (non-binding fraction) and a smaller second peak eluted later (titanium-binding fraction). Both peaks were analyzed by SDS polyacrylamide gel electrophoresis. Stains-all staining which preferentially identifies phospho-proteins revealed that the first peak contained no positively stained band, while the second peak showed 4 or 5 distinctive bands indicative of bone phosphoproteins. To investigate the biological functions of the titanium-binding bone proteins (TiBP), we implanted them into calvaria of rats, combined with titanium web (TW), a highly porous titanium scaffold of thin titanium-fibers. Bone TiBP induced significantly enhanced bone formation, and new bone appeared connected directly to titanium fibers, accompanied by active blood vessel formations. Control TW alone did not induce bone formation within the titanium framework. These results demonstrate that the bone titanium-binding proteins include phosphoproteins which enhance bone formation when implanted into bone with titanium.
\end{abstract}

Keywords: Bone phosphoprotein, titanium, bone formation, chromatography

\footnotetext{
*Address for correspondence: Yoshinori Kuboki, Professor Emeritus, Graduate School of Dental Medicine, Hokkaido University, N-13, W-7, Kita-ku, Sapporo, 060-8596, Japan. Tel.: +81 11706 4251; Fax: +81 11706 4251; E-mail: kuboki@den.hokudai.ac.jp.

** Yoshinori Kuboki and Toshitake Furusawa contributed equally to this study.
} 


\section{Introduction}

The biochemical mechanism behind the osteoconductivity of titanium has not been fully elucidated, in spite of worldwide clinical application of this characteristic feature of titanium [1-4]. We hypothesized that one of the essential mechanisms may reside in the interaction between certain proteins in the host tissues and the implanted titanium. We considered that the first groups of molecules which titanium (more precisely titanium oxides) encounters in bone tissues are serum proteins and ECM (extracellular matrix) components. To test this hypothesis we developed a titanium chromatography system involving a column packed with titanium beads. It was found that certain phosphoproteins including $\alpha$-and $\beta$-casein, phosvitin and dentin phosphoprotein (phosphophoryn) have selective binding ability to titanium, while most of the common proteins such as serum albumin and lysozyme do not [5].

It is well established that bone matrix is rich in various phosphoproteins, most of which are considered to play important roles in mineralization, growth and differentiation of bone cells although their functions are not fully elucidated [6-8]. Therefore the next question to be asked was whether titanium can bind with these phosphoproteins in bone or not. We hypothesized that implanted titanium bonding to bone is mediated by some of the bone phosphoproteins, if they are shown selectively to bind to it. Thus, the purpose of this investigation was to isolate and characterize the titanium-binding proteins in bovine bone as the first step to test this hypothesis.

\section{Materials and methods}

\subsection{Extraction of bone proteins}

Total soluble components of bone matrix were extracted by a modified method reported previously [9-11]. Briefly, fresh metatarsal bones of bovine were cleaned of soft tissues and pulverized in liquid nitrogen to obtain bone powders. Powders were decalcified with $0.01 \mathrm{M} \mathrm{HCl}$, keeping $\mathrm{pH} 2$ by constant addition of $6 \mathrm{M} \mathrm{HCl}$ and extracted with $2 \mathrm{M}$ urea/phosphate buffered saline (PBS).

\subsection{Chromatography}

Pure titanium beads with an average diameter $45 \mu \mathrm{m}$ were obtained commercially (Osaka Titanium Technologies, Co., Japan). Finer particles were carefully removed by repeated decantation from the suspension in distilled water. The beads were packed into a commercial glass chromatography column (XK16/20 GE Health Care, Tokyo, Japan) to obtain a bed volume of $16 \times 50 \mathrm{~mm}$. The urea extract was directly charged onto a chromatographic column packed with titanium beads, which was first eluted with $2 \mathrm{M}$ urea/phosphate buffered saline (PBS) followed by a gradient system between $2 \mathrm{M}$ urea/PBS and $2 \mathrm{M}$ urea/25 mM NaOH , each $100 \mathrm{ml}$, at a flow rate of $180 \mathrm{ml} / \mathrm{h}$. Elution was monitored by an automatic UV monitor system at $254 \mathrm{~nm}$ (AC 5100, Atoo Co., Japan).

\subsection{Polyacrylamide gel electrophoresis}

SDS-gel electrophoretic profiles were obtained from the first and the second peak from titanium chromatography, which were desalted by dialysis against distilled water at $5^{\circ} \mathrm{C}$ and lyophilized. Thirty $\mu \mathrm{g}$ of each sample was dissolved in SDS sample buffer and applied to a $15 \%$ polyacrylamide gel containing $0.1 \%$ SDS or a pre-cast gradient gel (5-20\%) (Pagel-compact, Atoo Co., Japan) with pre-stained standard molecular weight markers (Apro Life Science Co., Japan). The gel was stained with $0.0025 \%$ Stains-all [12]. 


\subsection{Implantation into rat calvaria}

Twenty pieces $(2 \times 3 \times 3 \mathrm{~mm}$ and approximately $8 \mathrm{mg}$ each) of titanium webs (TW) were immersed in a solution $(1 \mathrm{mg} / 500 \mu \mathrm{l})$ of titanium-binding bone proteins (TiBP). The TW/TiBP composites were air dried in a clean room and stored at $5^{\circ} \mathrm{C}$. Eight-weeks-old male rats (Wistar, 200-230 g) were anesthetized with pentobarbital sodium $(3.6 \mathrm{mg} / 100 \mathrm{~g}$ body weight, Nembutal R, Dainippon Sumitomo Pharma, Japan). The TW/TiBP composites were divided into two groups. One group was implanted on the temporal bone and beneath the periosteum. Another group was implanted into the holes created by diamond bur and trepan bur. After implantation, periostea and skin were carefully placed back and sutured. The protocol of the animal experiments was approved by the Committee for Animal Experiments in Kanagawa Dental College. It was carried out in accordance with guidelines proposed by the Institutional Animal Care and Use Committee. One piece of either the TW/TiBP composite or TW alone was implanted into one rat calvaria. After one month, the samples were removed for examination as described previously [4]. Parts of samples were retrieved after one week to see early responses.

\subsection{Histological observation}

Excised pieces of cranial bone, which contained implant, were fixed in $10 \%$ neutral formaldehyde, embedded in polyester resin (Rigolac, Oken, Japan), cut and polished into $80 \mu \mathrm{m}$ section by Maruto Systems (Crystal Cutter Nova MC-415Y and Speed Rap ML-521-d). Sections were stained with Cole's hematoxylin and eosin and analyzed histologically [4].

\section{Results}

\subsection{Adsorption of bone proteins on titanium column}

The results of the titanium chromatography of the $2 \mathrm{M}$ urea extract of bovine bone are shown in Fig. 1 . Most of the proteins applied to the column eluted in the breakthrough fraction, indicating that they were not adsorbed to the titanium. However, a smaller second peak was observed after elution with $25 \mathrm{mM}$ $\mathrm{NaOH}$, indicating that this protein fraction adsorbed to titanium with a certain affinity at neutral $\mathrm{pH}$, and was released at alkaline $\mathrm{pH}$.

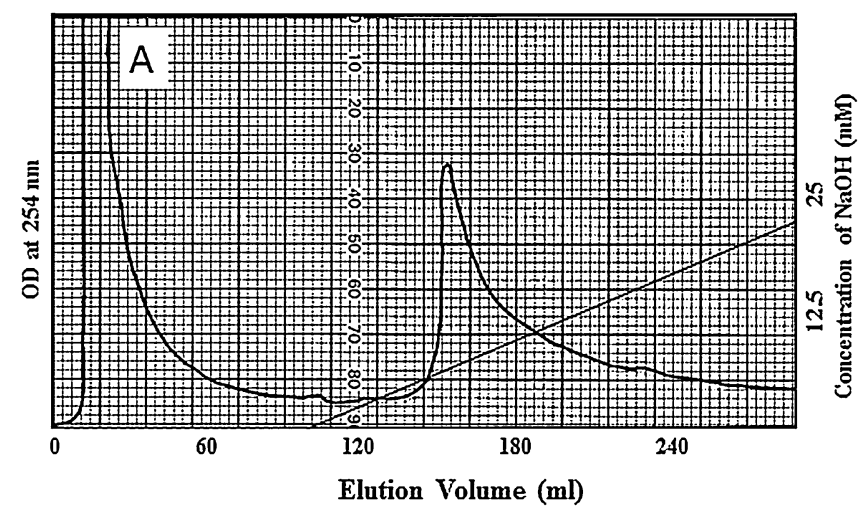

Fig. 1. Chromatogram of $2 \mathrm{M}$ urea extract of bone by a column packed with titanium beads. 


\subsection{Characterization of the titanium binding proteins from bone}

As shown in Fig. 2, the $15 \%$ gel electrophoretic profiles of the peak 2 from the chromatography of bone extract showed $4-5$ bands in the profile, which were stained by Steins-all. The molecular weights of these bands ranged near or larger than that of bovine serum albumin $(66 \mathrm{kDa})$, and much larger than $\beta$-casein $(24 \mathrm{kDa})$. Further characterization by electrophoresis in a gradient gel (5-20\%) and Stains-all staining (Fig. 3) showed that Peak 1 (indicated as Peak 1 in Fig. 3) was not stained by this method, while the $2 \mathrm{M}$ urea extract and Peak 2 (indicated as U-ext and Peak 2 in Fig. 3, respectively) were positively stained. Two lanes of Peak 2 were from different lots of the same methods of preparation. In both lanes, 4 or 5 bands were discernible as positive in Stain-all staining, though the resolution was still broad. Considering the absence of staining in the peak 1 lane, it was clearly shown that titanium

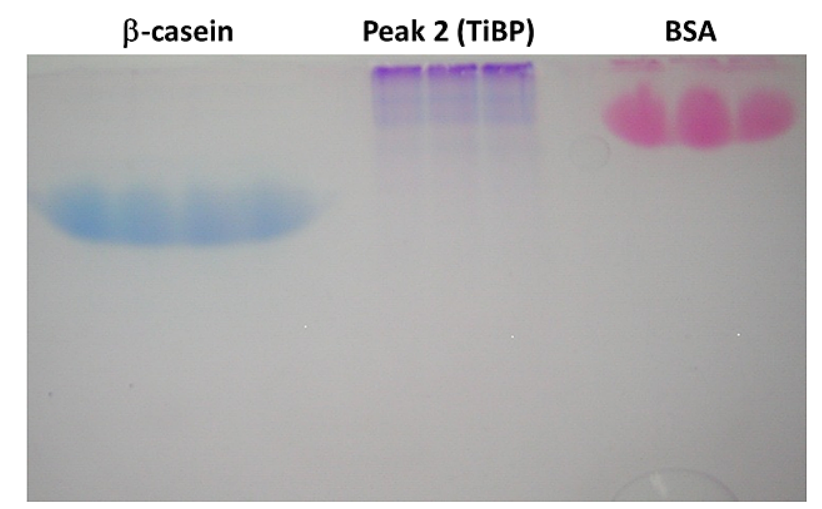

Fig. 2. SDS electrophoretic pattern in $15 \%$ gel of the second peak (titanium binding protein, TiBP), together with $\beta$-casein $(24 \mathrm{kDa})$ and bovine serum albumin $(66 \mathrm{kDa})$, stained with Stains-all, a staining method to stain phosphoprotein. (Colors are visible in the online version of the article; http://dx.doi.org/10.3233/BME-140958.)

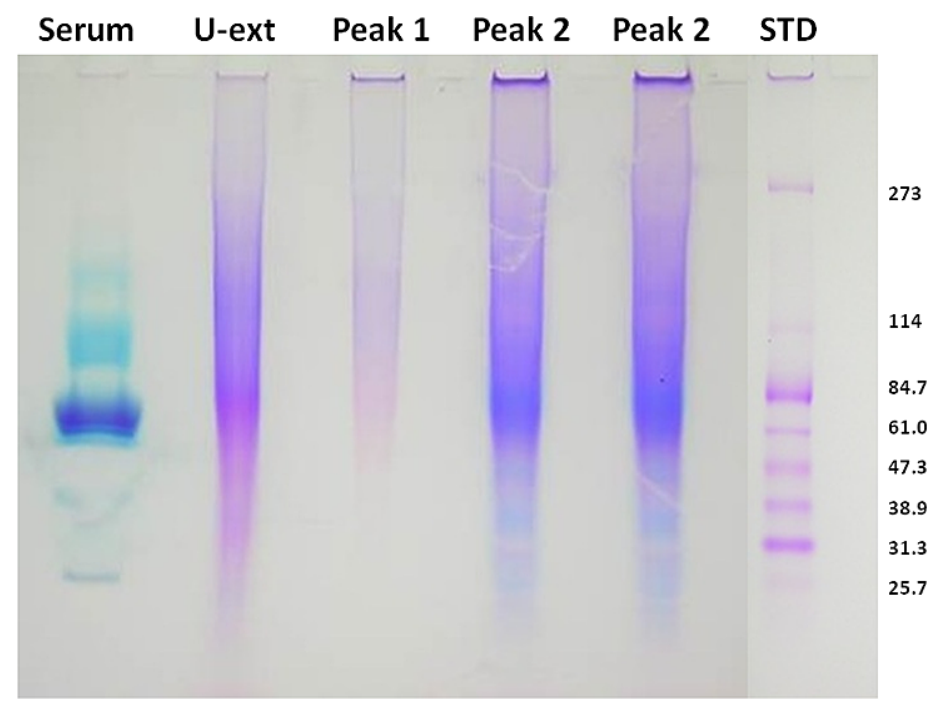

Fig. 3. SDS gel electrophoretic pattern (5-20\% gradient gel) of $2 \mathrm{M}$ urea extract (U-ext), peak 1 and peak 2 from titanium chromatography, together with bovine serum (Serum) and molecular markers (STD) stained with Stains-all. (Colors are visible in the online version of the article; http://dx.doi.org/10.3233/BME-140958.) 
chromatography concentrate the Stains-all positive substances, most probably phosphoproteins or at least acidic proteins. Bovine serum was stained in different color from phospho-proteins in this staining method, as already reported [12].

\subsection{Enhancement of bone formation in the titanium/phosphoprotein composites}

Histological observation of TW (titanium web) control and TW/TiBP composites retrieved one month after implantation are shown in Fig. 4(A-F). It can be seen that implantation of TW alone (Fig. 4(A)) induced scarcely new bone formation, although the condensation of collagen fibers are observed locally inside the TW framework (Fig. 4(B)). In contrast, TW/TiBP composites induced active new bone formation within the framework of TW (Fig. 4(C-F)).

At lower magnification (Fig. 4(C)), active bone formations in the central area of TW, together with partial resorption of temporal bone underneath the TW were observed. In enlarged images (Fig. 4(D) and (E)), many cross sections of blood vessels accompanied by endothelial cells (indicated by arrows) were seen near the center of the new bone formation. More prominent signs of active bone formation were revealed at the highest magnification (Fig. 4(F)), in that the form of flattened osteoblasts clearly
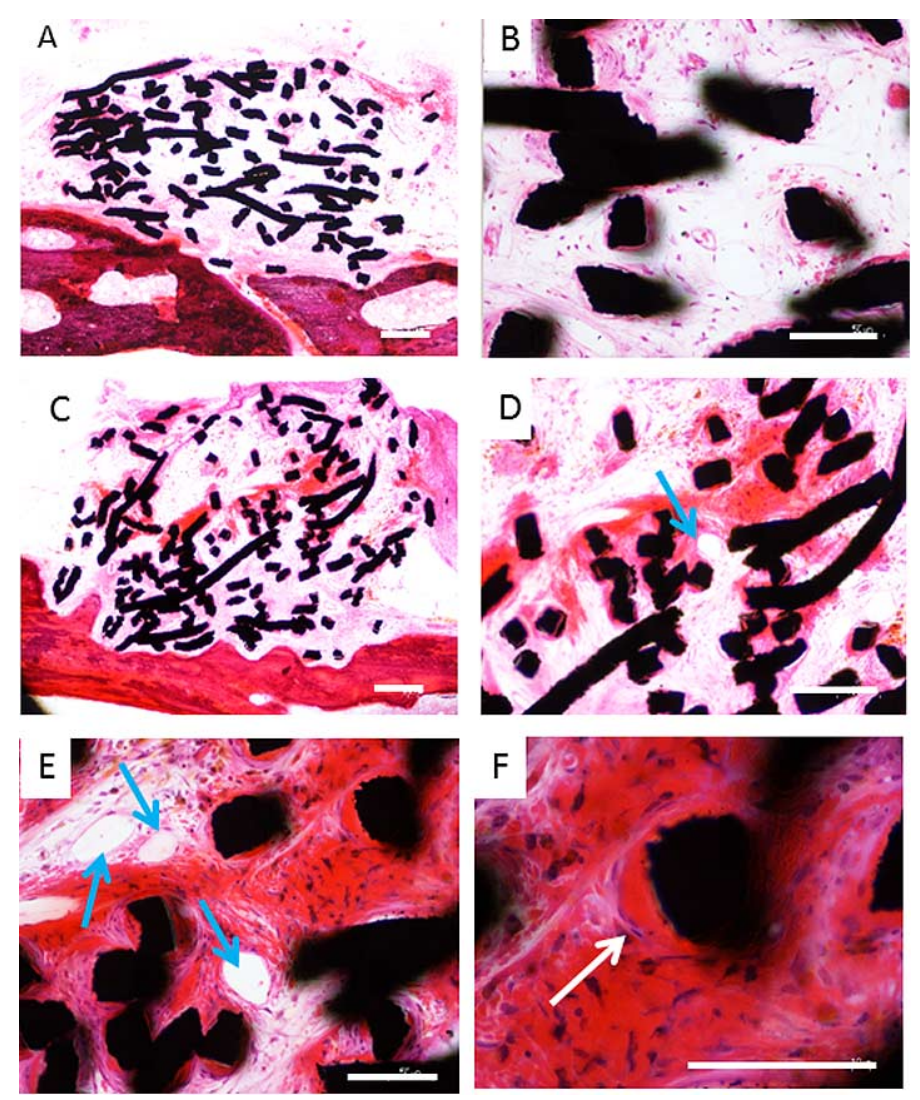

Fig. 4. Histological observation of the TW ((A) and (B)) and TW/TiBP (C-F), which were implanted between periosteum and dura matar of rat calvaria and retrieved after 4 weeks. Bars in (A), (C) and (D) indicate $200 \mu \mathrm{m}$, and (B), (E) and (F) $-100 \mu \mathrm{m}$. Blue arrows in (D) and (E) indicate blood vessels. The white arrow in Fig. 3(F) indicates active osteoblasts on the surface of newly-formed bone. (The colors are visible in the online version of the article; http://dx.doi.org/10.3233/BME-140958.) 
attached to the newly formed bone mass surrounding the titanium fibers. Another notable feature of bone formation in Fig. 4(F) is that all the newly-formed bone appeared to be closely attached to the surface of the titanium fibers, which is a plausible but rather rare observation in titanium-related osteogenesis.

In order to observe early reactions of the tissue against TW and TW/TiBP composites, they were implanted within the holes in temporal bone and retrieved after 1 week. In the control experiment of TW alone (Fig. 5(A)-(D)), no definite bone structure was found throughout the TW frame in lower magnification (A), but in enlarged views some bone-like structures were seen in the border areas between TW and existed bone. Contrastingly, in the TW/TiBP composites, definite mass of cells was observed in the middle area of TW frame (Fig. 5(E) and (F)). This structure seemed to be a highly condensed immature
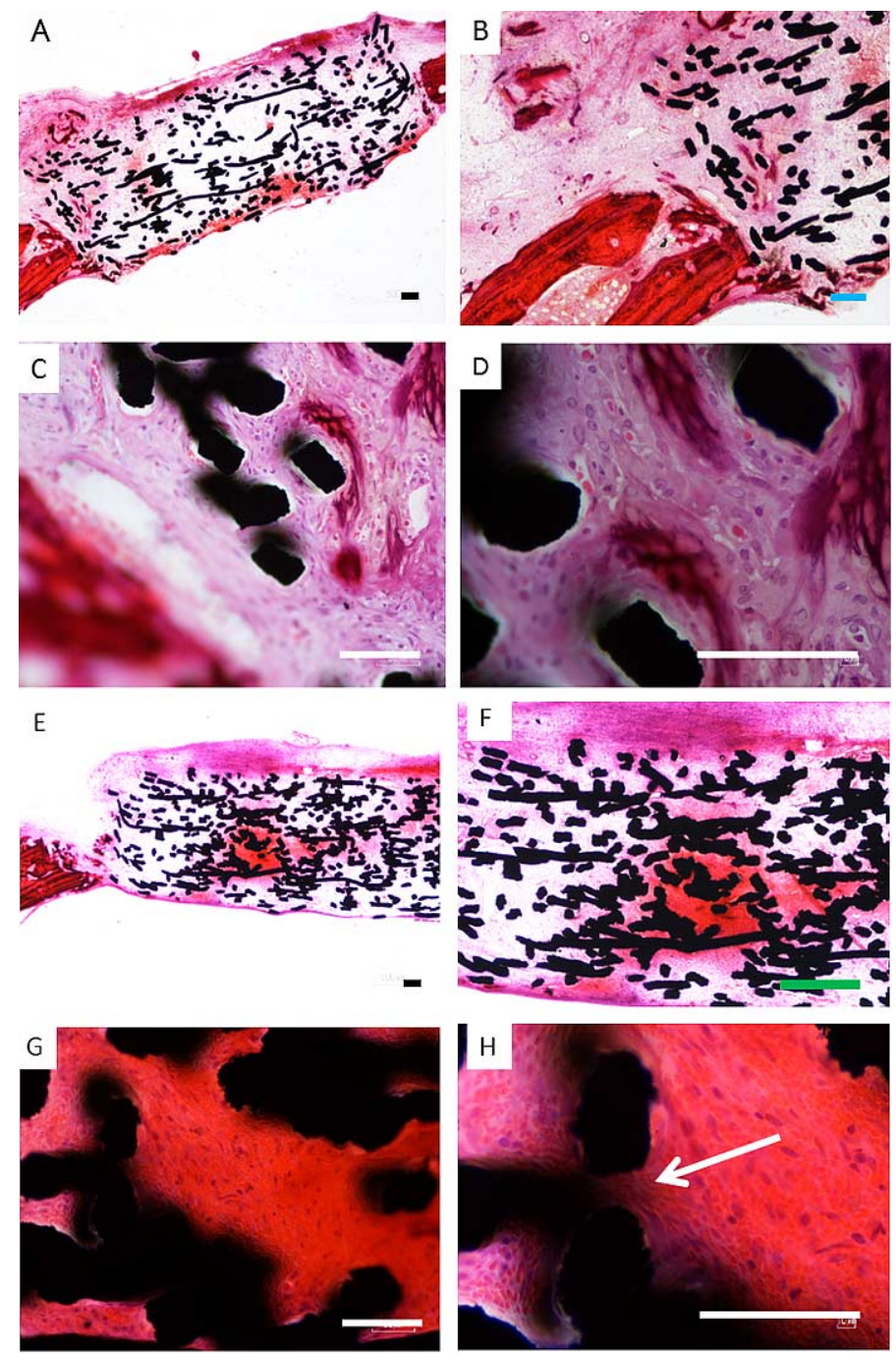

Fig. 5. Histological observation of the TW (A-D) and TW/TiBP composites (E-H), which were implanted into the holes created in rat calvaria between the periosteum and dura mater and retrieved after one week. Bars in (C), (D), (G) and (H) indicate $100 \mu \mathrm{m}$, and (A), (B) and (E), (F) - $200 \mu \mathrm{m}$. Blue arrows in (D) and (E) indicate blood vessels. The white arrow in (F) indicates active osteoblasts on the surface of newly-formed bone. (The colors are visible in the online version of the article; http://dx.doi.org/10.3233/BME-140958.) 


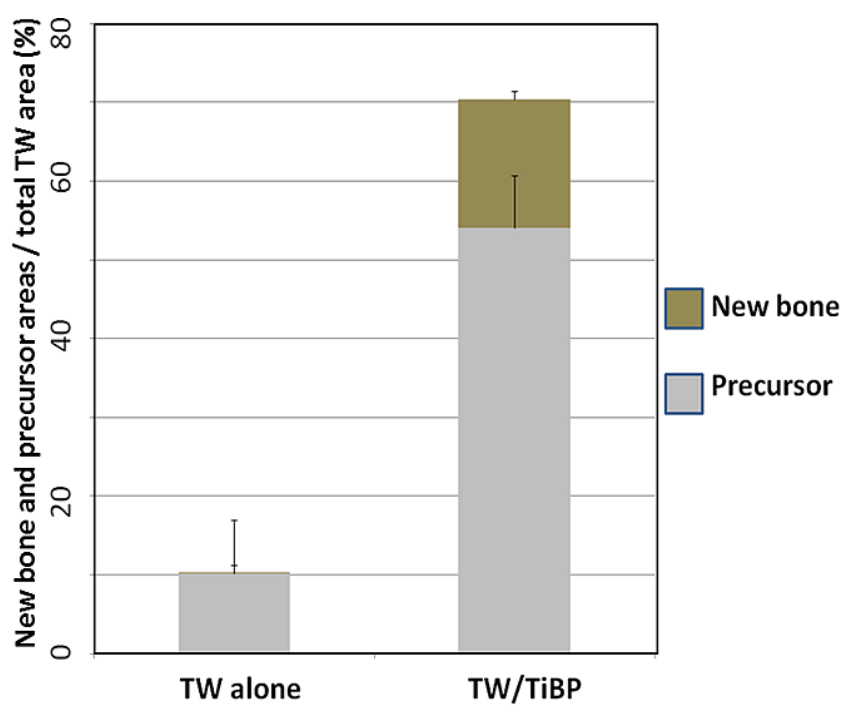

Fig. 6. Comparison of the amounts of bone formation in the scaffold of titanium web (TW) with and without titanium binding protein (TiBP). The areas of TW occupied by newly-formed mature bone and the precursor of bone were measured, and their ratios to the total TW areas were expressed in percent. The ratios were compared between TW alone and TW combined with TiBP (TW/TiBP). (Colors are visible in the online version of the article; http://dx.doi.org/10.3233/BME-140958.)

cells accompanied by rich intercellular matrices (indicated by an arrow Fig. 5(H)). Interestingly, the concentration of cells became much higher in the area near the surface of titanium fibers, seemingly directed by a chemo-attracting function of the titanium surface. Although the clear new bone was observed, tissue interaction by TW/TiBP was quite different from TW alone.

By detailed histological observation of the TW and TW/TiBP samples retrieved after one month (Fig. 4), the bone areas were divided into two areas: one is the precursor tissues of bone or fibrous bone, where the matrix accumulation is considerably high with rich vasculature, but still looser than that of mature bone tissues. The other is the mature bone with compact matrices, often surrounded by flatted osteoblasts. The ratios (\%) of areas occupied by the bone precursors and mature bone to the total areas of TW or TW/TIBP samples were calculated by Nis-Elements, Documentation (Nikon, Japan) in five different sections of the retrieved implants after one month (Fig. 6). Results indicated that the sum amounts of mature bone and its precursor in TW/TiBP is 7, precursor 5.2 and mature bone, more than 320 times higher than those in TW, indicating clear enhanced effect of TiBP on bone formation.

\section{Discussion}

Titanium beads chromatography separated the extract of bone proteins into non-binding breakthrough fraction (peak 1) and titanium binding fraction (peak 2) (Fig. 1). The results are similar to previously reported observation on common phosphoproteins, including phosvitin, $\alpha$ - and $\beta$-caseins [1]. Nonphosphorylated proteins such as serum albumin, lysozyme, fibrinogen and dephosphorylated phosvitin did not bind to the titanium column. Together with the positive results with the Stains-all staining method, they strongly suggested that TiBPs from bone most probably contain bone phosphoproteins.

Recently, growing attention has been directed towards the interaction between titanium, or more precisely, titanium oxides and phosphorylated peptides or proteins, particularly in the field of proteomics 
[13-15]. In spite of numerous reports, there are only a few studies on the application of titanium for the isolation and purification of proteins from animal tissues. To our knowledge, this paper is the first to isolate and characterize bone phosphoproteins by use of this interaction, though the definite characterization of individual bone phosphoproteins in TiBP are still to be done. Further purification of TiBP by using titanium or other chromatographic tools will clearly identify the components of bone TiBP.

Figures 4 and 5 demonstrate the eminent ability of bone TiBP to enhance bone formation when implanted with a titanium scaffold. So far, no single or a group of matrix proteins that has such enhancing effect when combined and implanted with titanium have been reported. There is still a possibility that the trace amount of cytokines in bone tissue, such as bone morphogenetic proteins, and insulin-like growth factors (IGFs) [8-11] were co-purified in the TiBP. If it is the case, titanium chromatography will provide a very useful tool in the purification of these cytokines. And at the same time, our hypothesis of "phosphoprotein mediation" will obtain an additional support. However, no evidence or suggestion has been reported on the affinity of cytokine of any kinds with titanium.

Three points can be noted concerning the histological observations of bone formation around the TiBP/TW. Firstly, bone formation occurred adjacent to the titanium fibers (Figs 4(E) and (F) and 5(G) and $(\mathrm{H})$ ), indicative of osteoconductivity. In addition, observation of condensation of cells in the early stage (Fig. 5(G) and (H)) strongly supports the chemo-attractant activity of the TiBP coated surface. Secondly, there are many blood vessels (Fig. 4(D) and (E)), which are important characteristic sign or requisite for new bone formation. Thirdly, flattened osteoblasts, which are typical of active bone formation, are clearly observed (Fig. 4(F)).

We chose a titanium web (abbreviated as TW) for the experiment, since we have developed this as the geometrically reasonable scaffold and proved to be highly effective for bone reconstruction $[4,16,17]$. TW is an unwoven mesh composed of thin titanium fibers, whose individual cross-section is square with widths of $50-80 \mu \mathrm{m}$, due to the manufacturing method. With its large surface area and the ideal size of inter-fibril space of $200-500 \mu \mathrm{m}$ for bone formation, TW may be the best carrier for TiBP. But the other forms of titanium materials are applicable for the carriers of TiBP. The bone TiBP is a mixture containing several phosphoproteins and may express mixed functions which include cell-migration, adhesion and regulation of growth and differentiation. Since this study showed the total function of TiBP is to enhance bone formation, we assume that TW/TiBP is a good model to study the stabilization mechanism of titanium devices in bone.

Recent studies on the non-collagenous matrix protein revealed that there are at least four different phosphoproteins which are categorized as members of a family known as SIBLINGs (small integrinbinding ligand N-linked glycoproteins) [6-8]. These are: osteopontin (OPN), bone sialoprotein (BSP), dentin matrix protein 1 (DMP1) and matrix extracellular phosphoglycoprotein (MEPE). These phosphoproteins were considered to have at least cell adhesive functions through their integrin binding sequence RGD [7,8], and the functions in mineralization [6]. However, the effects of the SIBLINGs on bone formation have not been clearly reported $[7,8]$. Therefore the mechanism of the remarkable enhancement of bone formation by TiBP is worth considering.

We propose that these proteins will express their bone enhancing ability only when they are bound with titanium substrates. The possible mechanism consisted of following three steps. First, bone phosphoproteins are immobilized on the surface of titanium, where they can promote cell adhesion. Second, osteoblasts or the precursors of osteoblasts attach to the cell-adhesive proteins proliferate and then differentiate. Thirdly, abundant phosphate groups of the immobilized phosphoproteins may provide environments feasible to mineralization of newly synthesized collagenous extracellular matrix. This sequence of events may occur in the actual titanium implantation clinically. Thus our hypothesis has been at least 
partially verified that the active bone formation around the titanium implants may be mediated by interaction between titanium and matrix proteins, possibly phosphoproteins.

\section{Conclusions}

In this study, it was shown for the first time that soluble bone proteins were separated into two groups: one consisting of proteins that are not bound to titanium (peak 1) and the other consisting of titanium-binding proteins (peak 2) by a chromatography column packed with titanium beads. The titanium-binding proteins were stained with Stains-all indicating that they contained bone phosphoproteins, while the non-binding proteins were not, indicating they did not contain bone phosphoproteins. It was further found that titanium-binding proteins showed a definite enhancement of bone formation when they were implanted into rat calvaria, combined with a titanium scaffold. The results strongly suggest that these proteins may provide a biochemical mechanism of stabilization when the titanium devices implanted into bone, and also point to many clinical applications of this phenomenon.

\section{Acknowledgements}

This study was supported in part by a Grant-in-Aid for Challenging Exploratory Research from the Ministry of Education, Culture, Sports and Technology, Japan (23659907). Authors appreciate precious advice given by Professor Emeritus Arthur Veis, Northwestern University, USA.

\section{References}

[1] Y. Kuboki, T. Furusawa, M. Sato, Y. Sun, H. Unuma, R. Fujisawa, S. Abe, T. Akasaka, F. Watari, H. Takita and R. Sammons, Interaction between titanium and phosphoproteins revealed by chromatography column packed with titanium beads, Biomed. Mater. Eng. 22 (2012), 283-288.

[2] P.I. Branemark, U.R. Adell, U. Breine, B.O. Hansson, J. Lindstrom and A. Ohlsson, Intra-osseous anchorage of dental prostheses. I. Experimental studies, Scand. J. Plast. Reconstr. Surg. 3 (1969), 81-100.

[3] P.I. Branemark, U. Breine, O. Hallen, B.O. Hansson and J. Lindstrom, Repair of defects in mandible, Scand. J. Plast. Reconstr. Surg. 4 (1970), 100-108.

[4] Y. Kuboki, S. Iku, R. Yoshimoto, T. Kaku and H. Takita, Modification of titanium surfaces based on the principles of geometry of the artificial extracellular matrix (ECM), in: Surface Design and Modification of the Biomaterials for Clinical Application, Transworld Research Network, J. Tanaka, S. Itoh and G. Chen, eds, Kerala, India, 2008, pp. 1-27.

[5] D.M. Brunette, P. Tengvall, M. Textor and P. Thomsen, Titanium in Medicine, Springer, Berlin, 2001, pp. 457-947.

[6] H.G.A. Ramachandran, T. Dahl, S. George, D. Schultz, D. Cookson, A. Veis and A. George, Phosphorylation of phosphophoryn is crucial for its function as a mediator of biomineralization, J. Biol. Chem. 280 (2005), 33109-33114.

[7] A. Bellahcene, V. Castronovo, K.U. Ogbureke, L.W. Fisher and N.S. Fedarko, Small integrin-binding ligand N-linked glycoproteins (SIBLINGs): multifunctional proteins in cancer, Nat. Rev. Cancer 8 (2008), 212-226.

[8] B. Huang, Y. Sun, I. Maciejewska, D. Qin, T. Peng, B. McIntyre, J. Wygant, W.T. Butler and C. Qin, Distribution of SIBLING proteins in the organic and inorganic phases of rat dentin and bone, Eur. J. Oral Sci. 116 (2008), 104-112.

[9] Y. Kuboki, H. Takita, D. Kobayashi, E. Tsuruga, M. Inoue, M. Murata, N. Nagai, Y. Dohi and H. Ohgushi, BMP-induced osteogenesis on the surface of hydroxyapatite with geometrically feasible and nonfeasible structures: topology of osteogenesis, J. Biomed. Mater. Res. 39 (1998), 190-199.

[10] D. Kobayashi, M. Takita, M. Mizuno, Y. Totsuka and Y. Kuboki, Time-dependent expression of bone sialoprotein fragments in osteogenesis induced by bone morphogenetic protein, J. Biochem. 119 (1996), 475-481.

[11] Y. Kuboki, M. Sasaki, A. Saito, H. Takita and H. Kato, Regeneration of periodontal ligament and cementum by BMPapplied tissue engineering, Eur. J. Oral Sci. 106(Suppl. 1) (1998), 197-203.

[12] K.P. Campbell, D.H. MacLennan and A.O. Jorgensen, Staining of the $\mathrm{Ca}^{2+}$-binding proteins, calsequestrin, calmodulin, troponin C, and S-100, with the cationic carbocyanine dye "Stains-all", J. Biol. Chem. 258 (1983), 11267-11273. 
[13] B.S. Lee, G.D. Lasanthi, P. Jayathilaka, J.S. Huang and S. Gupta, Immobilized metal affinity electrophoresis: a novel method of capturing phosphoproteins by electrophoresis, J. Biomol. Tech. 19 (2008), 106-108.

[14] M.R. Larsen, T.E. Thingholm, O.N. Jensen, P. Roepstorff and T.J. Jorgensen, Highly selective enrichment of phosphorylated peptides from peptide mixtures using titanium dioxide microcolumns, Mol. Cell Proteomics 4 (2005), 873-886.

[15] M.W. Pinkse, S. Lemeer and A.J. Heck, A protocol on the use of titanium dioxide chromatography for phosphoproteomics, Meth. Mol. Biol. 753 (2011), 215-228.

[16] Y. Kuboki, R. Yoshimoto, H. Kato, D. Li, T Kaku, H. Takita et al., A new paradigm of titanium-bone bonding: creation of the collaboration zone between the both substances by use of 3-D titanium web, which is attached to the titanium bulk by vacuum sintering, Arch. Bioceram. Res. 5 (2005), 146-149.

[17] N. Ichinohe, Y. Kuboki and Y. Tabata, Bone regeneration using titanium nonwoven fabrics combined with fgf-2 release from gelatin hydrogel microspheres in rabbit skull defects, Tissue Eng. Part A 14 (2008), 1663-1671. 